\title{
Die Konjunkturkomponente der Schuldenbremse - Spielräume und Grenzen
}

\author{
Der Beitrag diskutiert den verfassungsrechtlichen Rahmen für eine Reform der \\ Konjunkturkomponente der grundgesetzlichen Schuldenbremse. Diese Komponente \\ ermöglicht es, konjunkturelle Entwicklungen bei der Bestimmung der zulässigen Höhe \\ der Staatsverschuldung zu berücksichtigen. Bei ihrer Ausgestaltung sind vielfältige \\ Abwägungen zur Bestimmung der konjunkturellen Normallage zu treffen, insbesondere im \\ Hinblick auf die Vereinbarkeit von Familie und Beruf, das Ausmaß der Teilzeitarbeit und das \\ Renteneintrittsalter. Diese Entscheidungen sollten von der parlamentarischen Gesetzgebung \\ getroffen werden, die dabei über einen erheblichen Gestaltungsspielraum verfügt.
}

\begin{abstract}
Die Ampelkoalition braucht Geld. Die Bewältigung der wirtschaftlichen Corona-Folgen, Investitionen zur Beschleunigung der Energiewende und der steigende Zuschussbedarf der Sozialversicherung werden in den nächsten Jahren erheblichen Finanzbedarf verursachen. So überrascht es nicht, dass die Suche nach Verschuldungsspielräumen zu einem bestimmenden Thema der Koalitionsverhandlungen und der diese begleitenden öffentlichen Diskussion geworden ist. Einen zentralen Prüfstein aller Überlegungen bildet dabei die - durch die Föderalismusreform II 2009 neu konzipierte und in dieser Form seit dem vergangenen Jahr vollständig auf Bund und Länder anwendbare - Schuldenbremse der Art. 109 Abs. 3, 115 Abs. 2 GG. Diese lässt insbesondere Konstruktionen über Sondervermögen kaum zu, weil nach der Neurege-

(c) Der/die Autor:in 2021. Open Access: Dieser Artikel wird unter der Creative Commons Namensnennung 4.0 International Lizenz veröffentlicht (creativecommons.org/licenses/by/4.0/deed.de).

Open Access wird durch die ZBW - Leibniz-Informationszentrum Wirtschaft gefördert.

* Der Beitrag beruht auf einem Gutachten im Auftrag des Dezernat Zukunft - Institut für Makrofinanzen.
\end{abstract}

Prof. Dr. Stefan Korioth ist Inhaber des Lehrstuhls für Öffentliches Recht, insbesondere Kirchenrecht sowie Deutsches Staats- und Verwaltungsrecht an der Ludwig-Maximilians-Universität München.

Dr. Michael W. Müller ist dort wissenschaftlicher Mitarbeiter. lung auch Nebenhaushalte unter das grundsätzliche Verschuldungsverbot fallen. ${ }^{1}$

Größere Spielräume verspricht deshalb ein anderer Ansatz, der auf eine Reform innerhalb der Schuldenbremse zielt. Nach Art. 109 Abs. 3 Satz 2, Art. 115 Abs. 2 Satz 3 GG enthält die Schuldenbremse eine sogenannte Konjunkturkomponente. Zulässig sind danach Regelungen zur im Auf- und Abschwung symmetrischen Berücksichtigung der Auswirkungen einer von der Normallage abweichenden konjunkturellen Entwicklung. Wie dieser Beitrag zeigen wird, geben weder das Grundgesetz noch die derzeitige Ausgestaltung der Konjunkturkomponente durch Gesetz und Rechtsverordnung ${ }^{2}$ die maßgeblichen Parameter zur Bestimmung der Normallage abschließend vor. Dies ist für sich genommen bedenklich, weil damit wesentliche Entscheidungen über die Zulässigkeit der Neuverschuldung derzeit auf Anwendungsebene zu treffen sind. Die parlamentarische Gesetzgebung ist deshalb ohnehin zu einer Konturierung der Konjunkturkomponente aufgerufen. Je nach Ausgestaltung der Regelungen könnten - unter Wahrung der hier darzustellenden verfassungsrechtlichen Grundentscheidungen - für den Fall einer negativen Abweichung der konjunkturellen Situation von der "Nor-

1 Deutscher Bundestag $(2009,7)$; vgl. zuletzt auch - unter Verweis auch auf die zum Grundgesetz entwickelten Maßstäbe - Hessischer Staatsgerichtshof, Urteil vom 27.10.2021, P.St. 2783, P.St. 2827, Rn. 158 ff. Vgl. auch „Mehr Fortschritt wagen“, Koalitionsvertrag zwischen SPD, Bündnis 90/Die Grünen und FDP, 2021 (im Folgenden: Koalitionsvertrag), S. 160: „Die Berücksichtigung der Sondervermögen in der Schuldenregel erfolgt künftig 1:1 in dem verfassungsrechtlich erforderlichen Umfang."

2 Vgl. § 5 Abs. 2 des Gesetzes zur Ausführung von Artikel 115 des Grundgesetzes (im Folgenden: G-115), § 2 Abs. 2 der Verordnung über das Verfahren zur Bestimmung der Konjunkturkomponente nach $\S 5$ des Artikel 115-Gesetzes (Art. 115-V). 
mallage" zusätzliche Verschuldungsspielräume entstehen (zu einem konkreten Vorschlag Schuster et al., 2021). ${ }^{3}$

\section{Verfassungsrechtliche Spielräume der Konjunkturkomponente}

Die Konjunkturkomponente der Schuldenbremse erfährt im Grundgesetz derzeit folgende Ausgestaltung: Art. 109 Abs. 3 Satz 2 GG erlaubt - als Ausnahme vom grundsätzlichen Neuverschuldungsverbot nach Art. 109 Abs. 3 Satz 1 GG - neben Sonderregelungen für Naturkatastrophen oder außergewöhnliche Notsituationen Regelungen „zur im Auf- und Abschwung symmetrischen Berücksichtigung der Auswirkungen einer von der Normallage abweichenden konjunkturellen Entwicklung“. Für die Bundesebene wiederholt Art. 115 Abs. 2 Satz 3 GG diese Vorgabe, Art. 115 Abs. 2 Satz 4 GG sieht für Belastungen, die einen Schwellenwert von 1,5 vom Hundert im Verhältnis zum nominalen Bruttoinlandsprodukt überschreiten, vor, dass diese konjunkturgerecht zurückgeführt werden müssen. Art. 115 Abs. 3 Satz 5 GG überträgt sodann die weitere Ausgestaltung, „insbesondere die Bereinigung der Einnahmen und Ausgaben um finanzielle Transaktionen und das Verfahren zur Berechnung der Obergrenze der jährlichen Nettokreditaufnahme unter Berücksichtigung der konjunkturellen Entwicklung auf der Grundlage eines Konjunkturbereinigungsverfahrens sowie die Kontrolle und den Ausgleich von Abweichungen der tatsächlichen Kreditaufnahme von der Regelgrenze“, der Bundesgesetzgebung.

Bei dieser Ausgestaltung bestehen erhebliche Spielräume: Der verfassungsändernde Gesetzgeber hat sich 2009 mit dem Begriff der Normallage (statt „Störungen des Gesamtwirtschaftlichen Gleichgewichts“) bewusst dafür entschieden, eine neue Maßstabbildung zu ermöglichen (Deutscher Bundestag, 2009; Seiler, 2009, 724; Schmidt, 2009, 1279; von Lewinski, 2012, § 5 G-115 Rn. 5). Den verfassungsrechtlich zwingenden Begriffskern dürfte lediglich ausmachen, dass eine "Normallage“ tatsächlich erreichbar sein muss und ihre konkrete Nichterreichung ausschließlich Folge der konkreten wirtschaftlichen Situation sein darf. Weil die Normallage von der realen Situation gerade abweicht, bedarf es immer einer Festlegung unter Einbeziehung von Wertungen (Heun, 2018, Art. 115 Rn. 33), die prognostischen Charakter aufweist (Mayer, 2011, 276; auch - zu Art. 115 GG a.F. - BVerfGE 119, 96, 140 f.). Die einfließenden Wertungen dürfen freilich nicht völlig unrealistisch sein, aus der Normallage darf nicht im Rahmen der Ausgestaltung eine „Ideallage“ werden (Lenz und Burgbacher, 2009, 2563; Seiler, 2009, 724). Auch wird

3 Zur Zielsetzung einer Evaluierung des Konjunkturbereinigungsverfahrens auch Koalitionsvertrag, S. 161. in der Literatur zurecht angenommen, dass der Begriff der Normallage „,auf einen Korridor“ deutet, „in dem nicht jede noch so kleine Abweichung zum Schuldenmachen“ ermächtigen soll (Heintzen, 2021, Art. 109 Rn. 42).

Ferner muss das Verfahren eine symmetrische Berücksichtigung von Auswirkungen vorsehen, d.h. Auswirkungen im Aufschwung müssen spiegelbildlich Eingang in die Berechnung der zulässigen Neuverschuldung finden wie solche im Abschwung. Dabei geht es zwar um die Ausgestaltung des Berechnungsverfahrens, nicht um das konkrete Ergebnis der zulässigen Neuverschuldung (vgl. Seiler, 2009, 724; Christ, 2009, 1334); mit der Regelung ist aber der „teleologische Leitgedanke“ verknüpft, durch die Ausgestaltung sicherzustellen, dass die erfassten Abweichungen von der Normallage die tatsächlichen konjunkturellen Schwankungen abbilden und somit ein mittel- bis langfristiger Ausgleich von Kreditaufnahme und Tilgung erzielt wird (Pünder, 2010, Art. 115 Rn. 97; Mayer, 2011, 278 f.; Seiler, 2009, 724). Schließlich ist im Gesamtkontext der Schuldenbremse die grundsätzliche Zielsetzung des dauerhaften Haushaltsausgleichs im Blick zu behalten. Als Ausnahme hierzu muss die Konjunkturkomponente so begrenzt bleiben, dass es nicht letztlich zu einer dauerhaft zulässigen Neuverschuldung kommt (Schiller, 2013, 104 f.).

\section{Geringe Regelungstiefe in Gesetz und Verordnung}

Der in Art. 115 Abs. 3 Satz 5 GG ausgesprochene Regelungsauftrag bleibt im Gesetz zu Art. 115 GG erstaunlich unerfüllt (schon Korioth, 2009, 732; Schiller, 2013, 106; Seiler, 2009, 724; Mayer, 2011, 282). § 5 Abs. 2 G-115 bestimmt, dass eine Abweichung von der konjunkturellen Normallage vorliegen soll, wenn eine Unter- oder Überlastung der gesamtwirtschaftlichen Produktionskapazitäten erwartet wird; dies definiert $\S 5$ Abs. 2 G-115 als Produktionslücke und stellt in Satz 2 fest, dass dies der Fall sei, wenn das auf der Grundlage eines Konjunkturbereinigungsverfahrens zu schätzende Produktionspotenzial vom erwarteten Bruttoinlandsprodukt für das Haushaltsjahr, für das der Haushalt aufgestellt wird, abweicht. Sodann bestimmt $\S 5$ Abs. 3 G-115, dass sich die Konjunkturkomponente als Produkt aus der Produktionslücke und der Budgetsensitivität ergibt, die angibt, wie sich die Einnahmen und Ausgaben des Bundes bei einer Veränderung des gesamtwirtschaftlichen Gleichgewichts verändern. Wie sich die Produktionskapazitäten im einzelnen zusammensetzen und ermitteln lassen, regelt das G-115 nicht. Dieses enthält in § 5 Abs. 4 G-115 eine Verordnungsermächtigung zugunsten des Bundesministeriums der Finanzen, das im Einvernehmen mit dem Bundesministerium für Wirtschaft und Energie die Einzelheiten des Verfahrens zur Bestimmung der Konjunkturkomponente für den Bund in Übereinstimmung mit dem 
im Rahmen des Europäischen Stabilitäts- und Wachstumspakts angewandten Konjunkturbereinigungsverfahren festlegen soll, wobei das Verfahren regelmäßig unter Berücksichtigung des Standes der Wissenschaft zu überprüfen und fortzuentwickeln ist.

Aber auch die Ausgestaltung in der Art. 115-V bleibt vage: Zwar werden in $\S 2$ der Verordnung die Begriffe Produktionslücke und Budgetelastizität definiert. ${ }^{4}$ Insbesondere die nach $\S 2$ Abs. 2 Art. 115-V heranzuziehende Produktionsfunktion wird aber nur in Ansätzen beschrieben, das genaue Verständnis der einzustellenden Faktoren geht aus der Vorschrift nicht hervor (Baumann und Schneider, 2010, 104). Das ist insbesondere vor dem Hintergrund bedenklich, dass das Konjunkturbereinigungsverfahren gemäß § 5 Abs. 4 G-115 im Rahmen des Verordnungserlasses unter Berücksichtigung des Standes der Wissenschaft zu überprüfen und fortzuentwickeln ist. Der derzeitigen Ausgestaltung nach $\S 2$ Abs. 2 Art. 115-V ist jedoch nicht zu entnehmen, inwieweit das angewandte Verfahren dem Stand der Wissenschaft entspricht, weil die maßgeblichen wissenschaftlichen Auseinandersetzungen zur Konjunkturkomponente gerade die Bestimmung der einzustellenden Faktoren sowie des Trends der totalen Faktorproduktivität betreffen (zur unzureichenden Erfüllung des Gesetzgebungsauftrags auch Seiler, 2009, 724; Wendt, 2018, Art. 115 GG Rn. 44).

Verfassungsrechtlich ist das sehr bedenklich (Seiler, 2009, 725; Kube, 2009, Art. 115 Rn. 164; Wendt, 2018, Art. 115 Rn. 43 f.). Art. 80 Abs. 1 Satz 2 GG gibt für den Erlass von Rechtsverordnungen die Maßgabe vor, dass Inhalt, Zweck und Ausmaß der erteilten Ermächtigung im (Parlaments-)Gesetz bestimmt sein müssen. Dies verlangt von der parlamentarischen Gesetzgebung, die wesentlichen Fragen selbst zu entscheiden, d. h. insbesondere

4 Nach § 2 Abs. 2 Art. 115-V wird zur Ermittlung der Produktionslücke als Differenz zwischen Bruttoinlandsprodukt und Produktionspotenzial (§ 5 Absatz 2 des Gesetzes) das Produktionspotenzial geschätzt, das dem bei Normalauslastung der Produktionsfaktoren erreichbaren Bruttoinlandsprodukt entspricht. Die Schätzung erfolgt in Übereinstimmung mit dem im Rahmen der Haushaltsüberwachung nach dem Europäischen Stabilitäts- und Wachstumspakt angewandten Verfahren mit Hilfe einer gesamtwirtschaftlichen Produktionsfunktion vom Typ Cobb-Douglas. Über die Produktionsfunktion ergibt sich das Produktionspotenzial als Kombination aus den normal ausgelasteten Produktionsfaktoren Arbeit und Kapitalstock, multipliziert mit dem Trend der totalen Faktorproduktivität als Maß für den technischen Fortschritt bei Normalauslastung. Gemäß Absatz 3 erfasst die Budgetsensitivität die konjunkturbedingte Veränderung des Finanzierungssaldos des Bundes in Relation zum Bruttoinlandsprodukt, wenn das Bruttoinlandsprodukt um 1\% vom Produktionspotenzial abweicht. Sie errechnet sich als die mit den Bundesanteilen an den konjunkturabhängigen Einnahmen und Ausgaben des gesamtstaatlichen Haushalts gewichtete Summe der Teilelastizitäten der gesamtstaatlichen Budgetsensitivität, die auch in dem Verfahren zur Haushaltsüberwachung nach dem Europäischen Stabilitäts- und Wachstumspakt verwendet wird. das Normprogramm selbst festzulegen (BVerfGE 150, 1, 99 f. mit weiteren Nachweisen). Davon kann im G-115 keine Rede sein. Im Rahmen der Schätzung des Produktionspotenzials müssen Wertungen, etwa zur Vereinbarkeit von Familie und Beruf, zur Teilzeit- und Vollbeschäftigung und zum Renteneintrittsalter getroffen werden (Seiler, 2009, 724; Siekmann, 2021, Art. 109 Rn. 75; Kube, 2009, Art. 115 Rn. 164). Im Ergebnis hängt von diesen Wertungen die Zulässigkeit einer Kreditaufnahme mindestens in Millionenhöhe ab (Schuster et al., 2021, 49 f.). Die gegenwärtige Ausgestaltung überlässt die Entscheidung über die hier einzustellenden Annahmen der Rechtsanwendung (Seiler, 2009, 724; Pünder, 2010, Art. 115 Rn. 106), die hierdurch letztlich die kreditermächtigende Gesetzgebung binden kann (von Lewinski, 2012, § 5 G-115 Rn. 8).

$\mathrm{Zu}$ einer hinreichenden Bestimmtheit verhilft den Vorschriften auch nicht der in $\S 5$ Abs. 4 G-115 enthaltene Verweis auf das im Rahmen des Europäischen Stabilitäts- und Wachstumspakts angewandte Konjunkturbereinigungsverfahren. Dieses Verfahren ist im Unionsrecht nicht rechtsförmig festgelegt und für die Mitgliedstaaten nicht verbindlich. ${ }^{5}$ Wegen seiner fehlenden rechtlichen Grundierung trägt es auch nicht zur Erfüllung des Transparenzziels der Wesentlichkeitstheorie bei (Seiler, 2009, 725). Schließlich geht $\S 5$ Abs. 4 G-115 auch selbst nicht von einer vollständigen unionsrechtlichen Determination aus: Anderenfalls wäre die vorgesehene Festlegung durch Rechtsverordnung gar nicht erforderlich und die Vorgabe einer Überprüfung und Fortentwicklung des Verfahrens durch den Verordnungsgeber ginge ins Leere.

\section{Reformperspektiven}

Es bleibt also festzuhalten: Die im Grundgesetz zwingend vorgegebene, aber kaum konturierte Konjunkturkomponente knüpft das G-115 an die Ausschöpfung der Produktionskapazitäten. In verfassungsrechtlich bedenklicher Weise werden die dort einzustellenden Faktoren aber erst auf Verordnungsebene benannt, die dabei jeweils zu treffenden Annahmen erfahren gar keine materiell-rechtliche Festlegung. Die parlamentarische Gesetzgebung soll-

5 Das Unionsrecht gibt mit der sogenannten „Maastricht-Defizitgrenze“ nur eine Obergrenze der jährlichen öffentlichen Neuverschuldung vor, wobei es Ausnahmen im Fall des Wirtschaftsabschwungs vorsieht; vgl. Verordnung (EG) Nr. 1467/97 (ABIEG Nr. L 209 v. 2.8.1997, S. 6 ff.) zuletzt geändert durch Verordnung (EU) Nr. 1177/2011 (ABIEU Nr. L 306 v. 23.11.2011, S. 33 ff.); Verordnung (EG) Nr. 1466/97 (ABIEG Nr. L 209 v. 2.8.1997, S. 1 ff.), zuletzt geändert durch Verordnung (EU) Nr. 1175/2011 (ABIEU Nr. L 306 v. 23.11.2011, S. 12 ff.); dazu auf Grundlage der 2009 gültigen Fassungen Christ (2009, 1337); Pünder (2010, Art. 115 Rn. 106). Die Ausgestaltung des Konjunkturbereinigungsverfahrens auf Europäischer Ebene erfolgt lediglich durch exekutive Festlegung in Form eines Code of Conduct, vgl. ECOFIN-Rat (2016); zum Ganzen auf Grundlage der 2009 gültigen Fassung Seiler (2009, 725). 
te hier nachbessern. Gleichzeitig aber hat sie erhebliche Spielräume, die für die Berechnung des Produktionspotenzials maßgeblichen Inputfaktoren zu modifizieren und - innerhalb des erläuterten verfassungsrechtlichen Rahmens sonstigen wirtschaftspolitischen Wertungen anzupassen.

So ist etwa das Abstellen auf eine historische Festschreibung, wie es der den Lohnanstieg nicht beschleunigenden Arbeitslosenquote (non-accelerating wage rate of unemployment, NAWRU) zugrunde liegt, keinesfalls zwingend. Angesichts des hypothetischen Charakters der Normallage liegt es vielmehr nahe, das insgesamt zur Verfügung stehende Potenzial der Volkswirtschaft zu ermitteln und deshalb von der Zielsetzung der Vollbeschäftigung auszugehen (vgl. etwa den Vorschlag von Schuster et al., 2021, 43 ff.). Das Grundgesetz gewährt zwar kein Recht auf Arbeit, jedoch lässt sich dem Sozialstaatsprinzip und den Grundrechten auf Berufsfreiheit und freie Entfaltung der Persönlichkeit der Verfassungsauftrag entnehmen, auf Vollbeschäftigung hinzuwirken (grundlegend Böckenförde, 1980; Spieker, 1980, 787). Auch hinsichtlich der Partizipationsrate von Frauen sowie der einzubeziehenden Arbeitsstunden je Arbeitskraft sprechen wesentliche Gesichtspunkte gegen eine rein statistische Fortschreibung auf Basis der vergangenen Jahre. Vielmehr wurden in Verwirklichung der verfassungsrechtlichen Aufträge zur Gleichstellung der Geschlechter und zum Schutz von Ehe und Familie in vielen Bereichen Maßnahmen zur Verbesserung der Vereinbarkeit von Familie und Beruf getroffen (vgl. Müller, 2021, 74). Es liegt nahe, die potenziellen Effekte dieser Maßnahmen auch in die Bestimmung der volkswirtschaftlichen Normallage einzubeziehen.

Entsprechende Anpassungen der Inputfaktoren könnten sich gerade vor dem Hintergrund der wissenschaftlichen Kritik an NAWRU und an der statistischen Fortschreibung von Partizipationsrate und Teilzeitarbeit (Heimberger et al., 2017, 883 ff.; Schuster et al., 2021, 9 ff. mit weiteren Nachweisen) als Fortentwicklung des Konjunkturbereinigungsverfahrens im Sinne des $\S 5$ Abs. 4 G-115 darstellen. Nach der bisherigen Ausgestaltung der Konjunkturkomponente könnten solche Anpassungen sogar auf Verordnungsebene erfolgen; aufgrund der dargestellten verfassungsrecht- lichen Vorgaben ist jedoch für eine Neuregelung durch die parlamentarische Gesetzgebung zu werben.

\section{Literatur}

Baumann, E. und J. Schneider (2010), Die neue Regel des Bundes, in: C. Kastrop et al. Hrsg.), Die neuen Schuldenregeln im Grundgesetz, 89-123.

Böckenförde, E. W. (1980), Was nützen soziale Grundrechte?, Frankfurter Allgemeine Zeitung, 11. Februar 1980, 11

Christ, J. (2009), Neue Schuldenregel für den Gesamtstaat: Instrument zur mittelfristigen Konsolidierung der Staatsfinanzen, Neue Zeitschrift für Verwaltungsrecht, 28(21), 1333-1339.

Fraktionen der CDU/CSU und SPD im Bundestag (2009), Entwurf eines Gesetzes zur Änderung des Grundgesetzes (Artikel 91c, 91d, 104b, 109, 109a, 115, 143d), Bundestags-Drucksache 16/12410.

ECOFIN-Rat (2016), Specifications on the implementation of the Stability and Growth Pact and Guidelines on the format and content of Stability and Convergence Programmes (Beschluss des ECOFIN-Rats vom 5.7.2016), https://ec.europa.eu/economy_finance/economic_governance/sgp/pdf/coc/code_of_conduct_en.pdf (17. November 2021).

Heimberger, P., J. Kapeller und B. Schütz (2017), The NAIRU determinants: What's structural about unemployment in Europe?, Journal of Policy Modelling, 39(5), 883-908.

Heintzen, M. (2021), Art. 109, in J.-A. Kämmerer und M. Kotzur (Hrsg.), von Münch/Kunig, Grundgesetz, 7. Aufl.

Heun, W. (2018), Art. 115, in H. Dreier (Hrsg.), Grundgesetz, 3. Aufl.

Korioth, S. (2009), Das neue Staatsschuldenrecht - zur zweiten Stufe der Föderalismusreform, Juristenzeitung, 64(14), 729-737.

Kube, H., Art. 115 (2009), in R. Scholz et al. (Hrsg.), Maunz/Dürig, Grundgesetz, 56. Ergänzungslieferung.

Lenz, C. und E. Burgbacher (2009), Die neue Schuldenbremse im Grundgesetz, Neue Juristische Wochenschrift, 62(35), 2561-2567.

Mayer, C. (2011) Greift die neue Schuldenbremse?, Archiv des öffentlichen Rechts, 136(2), 266-322.

Müller, M. W. (2021), Unbedingte Rechtsansprüche im Bildungsföderalismus, Zeitschrift für Gesetzgebung, 36(1), 64-84

Pünder, H. (2010), Art. 115, in W. Höfling (Hrsg.), Berliner Kommentar zum Grundgesetz, 30. Ergänzungslieferung.

Schiller, R. (2013), Die verfassungsrechtliche Begrenzung der Staatsverschuldung.

Schmidt, R. (2009), Die neue Schuldenregel und die weiteren Finanzthemen der zweiten Föderalismusreform, Deutsches Verwaltungsblatt 124(20), 1274-1288

Schuster, F., M. Krahé und P. Sigl-Glöckner (2021), Wird die Konjunkturkomponente der Schuldenbremse in ihrer heutigen Ausgestaltung ihrer Aufgabe noch gerecht? Analyse und ein Reformvorschlag.

Seiler, C. (2009), Konsolidierung der Staatsfinanzen mithilfe der neuen Schuldenregel, Juristenzeitung, 64(14), 721-728

Siekmann, H. (2021), Art. 109, in M. Sachs (Hrsg.), Grundgesetz, 8. Aufl.

Spieker, W. (1980), Das Recht auf Arbeit im demokratischen Sozialstaat, Gewerkschaftliche Monatshefte, 1980(12), 777-788.

von Lewinski, K. (2012), Artikel 115-Gesetz.

Wendt, R. (2018), Art. 115, in P. M. Huber und A. Voßkuhle (Hrsg.), von Mangoldt/Klein/Starck, Grundgesetz, 7. Aufl.

Title: The Cyclical Component of the Debt Brake - Leeway and Limits

Abstract: This article discusses the constitutional framework for reforming the cyclical component of the debt brake in Germany. This component takes cyclical developments into account when determining the permissible level of public debt. When designing the cyclical component, a variety of judgments must be made to determine the normal cyclical situation, especially with regard to the compatibility of family and work, the extent of part-time work and the retirement age. The parliamentary legislature, which enjoys considerable leeway, should make these decisions.

JEL Classification: H63, J18, J68 